\title{
"Galileo Galilei" (GG) a small satellite to test the equivalence principle of Galileo, Newton and Einstein
}

\author{
Anna M. Nobili • Gian Luca Comandi • Suresh Doravari • Donato Bramanti • \\ Rajeev Kumar • Francesco Maccarrone • Erseo Polacco • Slava G. Turyshev • \\ Michael Shao • John Lipa · Hansjoerg Dittus • Claus Laemmerzhal • Achim Peters • \\ Jurgen Mueller • C. S. Unnikrishnan · Ian W. Roxburgh • Alain Brillet • \\ Christian Marchal • Jun Luo • Jozef van der Ha • Vadim Milyukov • Valerio Iafolla • \\ David Lucchesi • Paolo Tortora • Paolo De Bernardis · Federico Palmonari • \\ Sergio Focardi · Dino Zanello • Salvatore Monaco • Giovanni Mengali • \\ Luciano Anselmo • Lorenzo Iorio • Zoran Knezevic
}

Received: 24 April 2008 / Accepted: 6 October 2008 / Published online: 8 January 2009 (C) Springer Science + Business Media B.V. 2008

\footnotetext{
A. M. Nobili $(\varangle)$ - S. Doravari · D. Bramanti · F. Maccarrone · E. Polacco · G. Mengali University of Pisa, Pisa, Italy e-mail: nobili@dm.unipi.it
}

A. M. Nobili · G. L. Comandi · S. Doravari · R. Kumar · F. Maccarrone ·

E. Polacco · D. Lucchesi · L. Iorio

INFN, Pisa, Italy

G. L. Comandi · P. Tortora - F. Palmonari · S. Focardi

University of Bologna, Bologna, Italy

S. G. Turyshev · M. Shao

JPL, Pasadena, CA, USA

J. Lipa

Stanford University, Stanford, CA, USA

H. Dittus · C. Laemmerzhal

University of Bremen, Bremen, Germany

\section{A. Peters}

Humboldt University of Berlin, Berlin, Germany

J. Mueller

Technical University of Munich, Munich, Germany

C. S. Unnikrishnan

Tata Institute of Fundamental Research, Mumbai, India

I. W. Roxburgh

QMW, London, UK
A. Brillet
CNRS/OCA, Nice, France 
Abstract "Galileo Galilei" (GG) is a small satellite designed to fly in low Earth orbit with the goal of testing the Equivalence Principle-which is at the basis of the General Theory of Relativity-to 1 part in $10^{17}$. If successful, it would improve current laboratory results by 4 orders of magnitude. A confirmation would strongly constrain theories; proof of violation is believed to lead to a scientific revolution. The experiment design allows it to be carried out at ambient temperature inside a small 1-axis stabilized satellite (250 kg total mass). GG is under investigation at Phase A-2 level by ASI (Agenzia Spaziale Italiana) at Thales Alenia Space in Torino, while a laboratory prototype (known as GGG) is operational at INFN laboratories in Pisa, supported by INFN (Istituto Nazionale di fisica Nucleare) and ASI. A final study report will be published in 2009 .

Keywords General relativity $\cdot$ Space physics $\cdot$ Experimental gravity

\section{Introduction}

Today physics stands at the threshold of major discoveries. Growing observational evidence points to the need for new physics. Despite the success of General Relativity and the Standard Model, our present understanding of the fundamental laws of physics has several shortcomings. Although recent progress is very encouraging, the search for a realistic theory of quantum gravity remains a challenge. This

C. Marchal

ONERA, Châtillon, France

J. Luo

Huazhong University, Wuhan, People's Republic of China

J. van der Ha

University of Kyushu, Fukuoka City, Japan

V. Milyukov

Moscow State University, Moscow, Russia

V. Iafolla

IFSI-INAF, Rome, Italy

P. De Bernardis · S. Monaco

University of Rome "La Sapienza", Rome, Italy

F. Palmonari · S. Focardi

INFN, Bologna, Italy

D. Zanello

INFN, Rome "La Sapienza”, Italy

L. Anselmo

CNR/CNUCE, Pisa, Italy

Z. Knezevic

Beograd Observatory, Belgrade, Serbia 
continued inability to merge gravity with quantum mechanics indicates that the pure tensor gravity of general relativity needs modification or augmentation. It is now believed that new physics is needed to resolve this issue, motivating new searches for experimental signatures of very small deviations from general relativity and especially testing the Equivalence Principle (EP). This is because theoretical models proposing new physics to solve the problems mentioned above typically involve new interactions, some of which could manifest as violations of the equivalence principle. Such a situation offers a unique opportunity for a space based experiment and, hopefully, a major breakthrough.

$\mathrm{EP}$ is at the foundation of Einstein's general theory of relativity; therefore testing this principle is very important. The composition-independence of acceleration rates of various masses toward the Earth can be tested to many orders of magnitude greater precision in space-based laboratories, down to levels where some models of the unified quantum theory of gravity, matter, and energy suggest a possible violation of this principle. Even a confirmation of no violation in such an experiment will be exceptionally valuable; as such observations are guide posts in our quest for a physical theory unifying gravity and the Standard Model of particle physics. The scientific goal of "Galileo Galilei"-GG small satellite mission is to test the EP to 1 part in $10^{17}$. This accuracy would improve the present limits on the violation of EP by 4 orders of magnitude and could lead to a major discovery.

The best EP tests so far, performed with rotating torsion balances, have demonstrated that for the experimental apparatus (in essence a differential accelerometer) to achieve high sensitivity to an EP violation signal, it should have two major properties: an extremely weak coupling of the test masses (for high sensitivity to differential accelerations), and a very high rotation rate (to reduce noise through signal frequency modulation). In the field of classical mechanics a system satisfying both such properties has been known to exist since the beginning of last century. It goes under the name of "supercritical rotor" and is also known to require two degrees of freedom. Indeed, the space environment makes a supercritical rotor to perform much better than on Earth for three reasons. Firstly, because absence of weight allows the test masses to be coupled much more weakly than on Earth, by several orders of magnitude. Secondly, because frequency modulation can be achieved by rotating the whole spacecraft without a motor (by simply exploiting conservation of angular momentum), thus eliminating altogether motor and bearings noise, otherwise unavoidable on Earth. Thirdly, because in space the rotation axis of the satellite/rotor is not affected by low frequency terrain tilts, as it happens in the lab where these tilts compete with an expected EP violation signal, thus limiting the sensitivity of the apparatus. These advantages add to the 3 orders of magnitude increase of the driving signal for the EP violation effect with respect to its value in torsion balance experiments on the ground.

GG is unique in that it incorporates such a conceptually new accelerometer design (instead of the usual 1D single axis one). This choice naturally leads to a spacecraft passively stabilized by one axis rotation, with no need for active control after initial set-up. Cylindrical symmetry and fast rotation of the GG satellite around the symmetry axis make many disturbances unimportant (e.g. radiometer, patch effects etc.), allowing the experiment to be carried out at ambient temperature. With such an optimized design, the total mass of GG at launch has been carefully estimated to be of $250 \mathrm{~kg}$ only, a pivotal fact to reduce the complexity, the risks and the cost of a 
space mission. Last but not the least, the 2D design of the accelerometer allows a full scale ground test to assess the difficulty of the task, thus providing confidence in the chances of the space mission to achieve its goal. This is regarded as very important for space missions in fundamental physics, because they require-in addition to a perfect spacecraft for space navigation-a very challenging physics experiment to be performed in remote with no chance of improving or modifying the hardware from Earth.

In order to test the Equivalence Principle to 1 part in $10^{17}$, GG must detect a $0.6 \mathrm{pm}$ relative displacement of the test masses at $1.75 \times 10^{-4} \mathrm{~Hz}$ (the GG orbital frequency) in 1 week integration time. The GGG prototype supported by INFN and ASI in Italy (an apparatus of the same size as required in space, with the same degrees of freedom and the same displacement sensor) has demonstrated long duration, continuous operation (up to 2 months) and has reached the capability to detect relative displacements of 1 nanometer (at a few $10^{-3} \mathrm{~Hz}$ ). This result has been obtained in the presence of noise from motor and bearings and from low frequency terrain tilts, which are all absent in space. All these limiting factors can-and will-be further reduced.

In this paper we focus on the scientific case (Why is a high accuracy test of the Equivalence Principle so important for today's physics?-Section 2) and then concentrate on a few crucial properties of the GG space experiment design which make it possible to aim at an extremely ambitious scientific goal with a small satellite (Section 3). Finally, we devote Subsection 3.1 to the GGG laboratory prototype, to demonstrate the closeness of its physical design to that of the instrument to fly in GG and the relevance of its performance to increase confidence in the success of the space mission.

\section{Testing the equivalence principle to very high accuracy: the scientific case}

General Theory of Relativity (GTR) and the Standard Model (SM) of particle physics, taken together, form our current view of the physical world. While the former governs physics in the macroscopic and cosmic scales the latter governs the physics of the microcosm. According to GTR, gravity is not a force but a manifestation of space-time curvature. The relation between space-time curvature and space-time content (mass-energy and momentum) being given by Einstein's field equations. The theory has been extensively tested and no astronomical observation or experimental test (the most accurate of which have been performed in space) has been found to deviate from its predictions. Thus it is the best description we have of gravitational phenomena that we observe in nature. The Standard Model of particle physics gives a unified formalism for the other three fundamental interactions (strong, weak and electromagnetic) between the fundamental particles that make up all matter. It is a quantum field theory which is consistent with both Quantum Mechanics and Special Theory of Relativity. To date, almost all experimental tests of the Standard Model have also agreed with its predictions.

However, merging these two very successful theories to form a single unified theory poses significant difficulties. While in SM particle fields are defined on a flat Minkowski space-time, GTR postulates a curved space-time which evolves with the motion of mass-energy. The definition of a gravitational field of a particle, whose 
position and momentum are governed by the Heisenberg Uncertainty Principle, is unclear. In addition quantum mechanics becomes inconsistent with GTR near singularities. Attempts at reconciling these theories often lead to a violation of the Equivalence Principle on which GTR is based. Therefore tests of the Equivalence Principle address a crucial problem which is at the heart of fundamental physics today.

In addition, the need to understand the nature of dark matter, the recent remarkable discoveries of observational cosmology and the puzzle of dark energy, all indicate that physics beyond the Standard Model and the General Theory of Relativity is needed. Invoked by most astronomers, dark matter probably consists of undiscovered elementary particles whose aggregation produces the gravitational pull capable of holding together galaxies and clusters of galaxies. It should account for more than $20 \%$ of the total mass in the universe but is not understood as yet. Dark energy is an even deeper mystery. Recent measurements show that the expansion of the universe is speeding up rather than slowing down, thus contradicting the fundamental idea that gravity is always attractive and calling for the presence of an unknown form of energy (the "dark energy") — whose gravity is repulsive and whose nature determines the evolution of the universe-which should contribute by about $70 \%$ to its total mass.

The major questions now being asked about the universe at its two extremes- the very large and the very small—appear to be inextricably intertwined.

The National Research Council of the US National Academies has appointed a specific "Committee on the Physics of the Universe" to investigate the subject and advise the major national research funding agencies. The results of the panel's work have been published in the book "Connecting Quarks with the Cosmos: Eleven Science Questions for the New Century", (National Academies press, 2003) [1].

The third of the 11 questions identified in the book is "Did Einstein Have the Last Word on Gravity?" and reads:

"Black holes are ubiquitous in the universe, and their intense gravity can be explored. The effects of strong gravity in the early universe have observable consequences. Einstein's theory should work as well in these situations as it does in the solar system. A complete theory of gravity should incorporate quantum effects-Einstein's theory of gravity does not-or explain why they are not relevant."

The last chapter of the book, under the title "Realizing the Opportunities", is devoted to giving recommendations as to how to proceed in order to answer the 11 questions identified. The recommendations focus on very large scientific projects; however, a specific Section called "Striking the Right Balance" reads (p. 162):

"In discussing the physics of the universe, one is naturally led to the extremes of scale - to the largest scales of the universe as a whole and to the smallest scales of elementary particles. Associated with this is a natural tendency to focus on the most extreme scale of scientific projects: the largest space observatories, the most energetic particle accelerators. However, our study of the physics of the universe repeatedly found instances where the key advances of the past or the most promising opportunities for the future come from work on a very different scale. Examples include laboratory experiments to test gravitational interac- 
tions, theoretical work and computer simulations to understand complex astrophysical phenomena, and small-scale detector development for future experiments. These examples are not intended to be exhaustive but to illustrate the need for a balanced program of research on the physics of the universe that provides opportunities for efforts that address the scientific questions but that do not necessarily fit within major program themes and their related large projects.

Two of our scientific questions-'Did Einstein have the last word on gravity?' and 'Are there additional space-time dimensions?'-are being addressed by a number of laboratory and solar-system experiments to test the gravitational interaction. Tests of the principle of equivalence using laboratory torsion balances and lunar laser ranging could constrain hypothetical weakly coupled particles with long or intermediate range. These experiments have reached the level of parts in $10^{13}$ and could be improved by another order of magnitude. Improvement by a factor of around $10^{5}$ could come from an equivalence principle test in space... null experimental results provide important constraints on existing theories, and a positive signal would make for a scientific revolution."

In addition to the "Committee on the Physics of the Universe", a Dark Energy Task Force (DETF) has been established in the USA by the Astronomy and Astrophysics Advisory Committee and the High Energy Physics Advisory Panel to advise the Department of Energy, NASA and the National Science Foundation on future dark energy research. In 2006 the DETF published its final report [2], where the Executive Summary begins as follows:

"Over the last several years scientists have accumulated conclusive evidence that the Universe is expanding ever more rapidly. Within the framework of the standard cosmological model, this implies that $70 \%$ of the universe is composed of a new, mysterious dark energy, which unlike any known form of matter or energy, counters the attractive force of gravity. Dark energy ranks as one of the most important discoveries in cosmology, with profound implications for astronomy, high-energy theory, general relativity, and string theory.

One possible explanation for dark energy may be Einstein's famous cosmological constant. Alternatively, dark energy may be an exotic form of matter called quintessence, or the acceleration of the Universe may even signify the breakdown of Einstein's Theory of General Relativity. With any of these options, there are significant implications for fundamental physics."

A few pages below, the Section of the Report on "Goals and Methodology for Studying Dark Energy" ends with the following sentence:

"Just as dark-energy science has far-reaching implications for other fields of physics, advances and discoveries in other fields of physics may point the way toward understanding the nature of dark energy; for instance, any observational evidence for modifications of General Relativity." 
The principle of equivalence has historically played a major role in the development of gravitation theory. It is possible to ascribe two conceptually different kinds of mass to a body: an inertial mass and a gravitational mass. The inertial mass is the proportionality factor between a force (any kind of force) applied to the body and the acceleration it acquires in response to it in an inertial laboratory. The gravitational mass is a measurement of the property of the body to attract gravitationally any other body (gravitational active mass), or to be gravitationally attracted by any other body (gravitational passive mass). Assuming the validity of the action-reaction principle (which leads to conclude that the center of mass of an isolated system must move with constant velocity in an inertial frame of reference) also implies that the gravitational passive and active mass of a body must be the same. The gravitational mass is the analog in a gravitational field, of the electric charge in an electric field-it can be viewed as a gravitational charge - and it has no apparent relation (in spite of the name) with the concept of inertial mass. Using Newton's law of gravitation to write the equation of motion of a body of inertial mass $m_{\mathrm{i}}$ and gravitational mass $m_{\mathrm{g}}$ in the field of a source body of gravitational mass $M_{\mathrm{g}}$ (for instance, the Earth), if $m_{\mathrm{i}} \propto m_{\mathrm{g}}$ the resulting acceleration is the same for all bodies. With the measured value of the gravitational constant $G$ and a proportionality factor $+1\left(m_{\mathrm{i}}=m_{\mathrm{g}}\right)$, the local acceleration of gravity on the surface of the Earth-the same for all bodies regardless of their mass and composition-amounts to about $9.8 \mathrm{~m} / \mathrm{s}^{2}$. This is the so called Universality of Free Fall (UFF). No such thing holds for all other fundamental forces of Nature. For instance, a proton and an electron do not have-in the same electric field - the same (in modulus) acceleration, because the inertial mass of the proton is much larger than the inertial mass of the electron and no proportionality holds between the inertial mass of a body and its electric charge.

Galileo was most probably the first one to provide experimental evidence for the UFF [3]. However, he was not aware of the law which rules the gravitational interaction. Therefore, he had no awareness either of the equivalence between inertial and gravitational mass, and of the link between this concept and his own experimental results on the UFF. The fact that the two concepts of inertial and gravitational mass refer in fact to the same physical quantity was first stated by Newton in the opening paragraph of the Principia [4]: "This quantity that I mean hereafter under the name of... mass... is known by the weight... for it is proportional to the weight as I have found by experiments on pendulums, very accurately made..."

At the beginning of the twentieth century, almost 300 years since Galileo's work, Einstein realized that because of the proportionality between the gravitational mass and the inertial mass, the effect of gravitation is locally equivalent to the effect of an accelerated frame and can be locally canceled. This is known as the Weak Equivalence Principle (WEP) which Einstein introduced in 1907 [5] as the "hypothesis of complete physical equivalence" between a gravitational field and an accelerated reference frame: in a freely falling system all masses fall equally fast, hence gravitational acceleration has no local dynamical effects. Any test mass located inside the famous Einstein elevator-falling with the local acceleration of gravity $g$ near the surface of the Earth-and zero initial velocity with respect to it, remains motionless for the time of fall. An observer inside Einstein elevator will not be able to tell, before hitting the ground, whether he is moving with an acceleration $\mathrm{g}$ in empty space, far away from all masses, or else he is falling in the vicinity of a body (the Earth) whose local gravitational acceleration is also $g$ (and in the same direction). 
Einstein's formulation of the Weak Equivalence Principle whereby the effect of gravity disappears in a freely falling reference frame, holds only locally. The elevator is free falling in the vicinity of the Earth, which amounts to saying that the height of fall is much smaller than the radius of the Earth. The cancellation of gravity in a freely falling frame holds locally for each frame, but the direction of free fall is not the same in all of them. Which is a direct consequence of the fact that the gravitational field of a body (like Earth) is non uniform, giving rise to the so called tidal forces between test particles whose centers of mass are not coincident. With the WEP Einstein has moved from Newton's concept of one global reference frame with gravitational forces and the UFF, to many free falling local frames without gravitational forces.

In his further development of the General theory of Relativity Einstein formulated what is known as the Einstein Equivalence Principle (EEP), which is an even more powerful and far reaching concept. EEP states the following (see e.g. [6]): (1) WEP is valid; (2) The outcome of any local non-gravitational experiment is independent of the velocity of the freely-falling reference frame in which it is performed (Local Lorentz Invariance); (3) The outcome of any local non-gravitational experiment is independent of where and when in the universe it is performed (Local Position Invariance). The Einstein Equivalence Principle-which assumes the weak one-is regarded as the "heart and soul" of the General Theory of Relativity because it is the validity of this "principle" to ensure the fact that in General Relativity the effects of gravity are replaced by a curved 4-dimensional space-time.

From an experimental point of view, a violation of the Universality of Free Fall would invalidate the Weak Equivalence Principle, hence the Einstein Equivalence Principle, thus placing a limit on the validity of the General Theory of Relativity itself. This is the physical motivation behind a continuing interest within the scientific community worldwide in performing more and more accurate experimental tests of the UFF_on Earth and hopefully also in space.

In an experiment to test UFF the observable physical quantity is the differential acceleration $\Delta \mathrm{a}$ of two test masses of different composition, relative to each other, while falling in the gravitational field of a source body with an average acceleration a (also referred to as the "driving acceleration"). A deviation from UFF is therefore quantified by the dimensionless parameter

$$
\eta=\frac{\Delta \mathrm{a}}{\mathrm{a}}
$$

The finding of a value $\eta \neq 0$ would disprove the UFF and indicate a violation of the Weak Equivalence Principle on which General Relativity ultimately relies. Instead, $\eta=0$ - as reported by all experiments so far-confirms the basic assumption of General Relativity. By writing the equations of motion of each individual test mass without assuming a priori the equivalence of their inertial and gravitational mass, the parameter $\eta$ given by Eq. 1 becomes

$$
\eta=\frac{2\left[\left(m_{\mathrm{g}} / m_{\mathrm{i}}\right)_{A}-\left(m_{\mathrm{g}} / m_{\mathrm{i}}\right)_{B}\right]}{\left[\left(m_{\mathrm{g}} / m_{\mathrm{i}}\right)_{A}+\left(m_{\mathrm{g}} / m_{\mathrm{i}}\right)_{B}\right]}
$$

where subscripts $A$ and $B$ refer to the individual test masses and allow them to be distinguished by their different composition. This parameter $\eta$ is also known as the Eötvös parameter and it has additional profound significance. 
The total mass-energy of a body can be expressed as the sum of many terms, corresponding to the energy of all the conceivable interactions and components: $m=\Sigma_{k} m_{k}$. For instance, at the atomic level, the rest mass contributes (as a fraction of the total) for $\cong 1$; the nuclear binding energy for $8 \times 10^{-3}$ (for light elements), the mass difference between neutron and proton for $1.4 \times 10^{-3}(A-Z) / A$ ( $A$ being the number of protons plus neutrons and $Z$ the number of protons in the nucleus), the electrostatic energy of repulsion in the nuclei for $6 \times 10^{-4} Z^{2} A^{-4 / 3}$, the mass of electrons for $5 \times 10^{-4} Z / A$, the antiparticles for $\cong 10^{-7}$, the weak interactions responsible of $\beta$ decay for $10^{-9}$ or less. For an extended spherical body of radius $R$ and (homogeneous) density $\rho$, the gravitational self-energy contributes by the fraction-4/5 $\pi \rho G R^{2} / c^{2}$. The conventional Eötvös parameter (Eq. 2) can therefore be generalized into:

$$
\eta_{k}=\frac{2\left[\left(m_{\mathrm{g}} / m_{\mathrm{i}}\right) A_{k}-\left(m_{\mathrm{g}} / m_{\mathrm{i}}\right) B_{k}\right]}{\left[\left(m_{\mathrm{g}} / m_{\mathrm{i}}\right) A_{k}+\left(m_{\mathrm{g}} / m_{\mathrm{i}}\right) B_{k}\right]}
$$

such that a non-zero value of $\eta_{k}$ would define the violation of equivalence between the inertial and gravitational mass-energy of the $k$-th type. From the point of view of conventional field theory, the verification of all these separate "Equivalence Principles" corresponds to a very peculiar coupling of each field to gravity; whether and why it should be so in all cases is a mystery.

It is apparent from Eq. 1 that-for any given experimental apparatus-the larger the driving acceleration, the more sensitive the UFF test (hence the EP test) that it provides. In a Galileo-type mass dropping experiment the driving acceleration is the gravitational acceleration of the Earth along the local vertical $\left(9.8 \mathrm{~m} / \mathrm{s}^{2}\right)$. If the test masses are suspended on a torsion balance the driving acceleration is $0.017 \mathrm{~m} / \mathrm{s}^{2}$ (at most) in the field of the Earth-directed along the North-South direction of the local horizontal plane-and $0.006 \mathrm{~m} / \mathrm{s}^{2}$ in the field of the Sun (with components along the North-South and East-West directions of the horizontal plane) (see e.g. [7]). Yet, the first experimental apparatus to provide very accurate EP tests (to $10^{-8}-10^{-9}$ ) was the torsion balance used by Eötvös at the turn of the twentieth century, and later on by his students [8] to detect an EP violation in the field of the Earth. This is because torsion balances are extremely sensitive; moreover, they are inherently differential instruments, and although in reality perfect rejection of common mode effects is impossible, the advantages of a differential instrument for detecting differential accelerations are enormous.

The next leap in sensitivity (to $10^{-11}-10^{-12}$ ) came in the 1960 s and early $1970 \mathrm{~s}$ $[9,10]$ using again a torsion balance but also recognizing that by taking the Sun as the source mass rather than the Earth, any differential effect on the test masses of the balance would be modulated by the $24 \mathrm{~h}$ rotation of the Earth on which the balance sits. Indeed, the modulation frequency should be as high as possible, in order to reduce $1 / f$ electronic noise. The best and most reliable results in EP testing (to about 1 part in $10^{12}$ and, as recently announced, to about a part in $10^{13}$ ) have been achieved by the "Eöt-Wash" group at the University of Seattle in a systematic series of experiments using torsion balances placed on a turntable which modulates the signal with a period down to about 20 minutes [11-13]. They have tested pairs of $\mathrm{Be}-\mathrm{Cu}$ and $\mathrm{Be}-\mathrm{Al}$ in the field of the Earth as well as the Sun [11], and test masses of Earth-like and Moon-like composition in the field of the Sun [12] to remove the 
ambiguity of lunar laser ranging tests of the equivalence principle for gravitational self-energy.

Despite the much larger driving acceleration, Galileo-type mass dropping tests of UFF have been unable to compete with rotating torsion balances [14]. The success of torsion balances relies on three main properties: (1) high sensitivity to differential accelerations; (2) long time duration of the experiment; (3) up-conversion of the signal (DC from the Earth and 24-h from the Sun) to higher frequency. Flying an instrument with these properties in low orbit around the Earth would add the only advantage of mass dropping, namely the very large driving acceleration from Earth. This fact alone would provide-assuming the same sensitivity to differential accelerations as achieved in ground tests-an improvement in $\eta$ by about 3 orders of magnitude. The difficulties related to running the experiment in space, with no direct access to it, can be compensated by exploiting those peculiarities of the space environment which are relevant for experiments to test UFF, most importantly, absence of weight and isolation of the satellite/experiment once in orbit. In Section 3 we shall see how absence of weight and system isolation can significantly contribute to improving the current best tests of EP by several orders of magnitude.

The difficulties of torsion balance tests explain the slow improvement of their results over the years and indicate that considerable progress beyond the current level is extremely hard to achieve. A new type of experiments based on interferometry of free falling cooled atoms is in preparation with the very ambitious goal of performing in the lab an EP to $10^{-15}$ and even to $10^{-17}$ sometime in the future [15]. So far a measurement of the local acceleration of falling atoms has been performed by [16] achieving $\Delta g / g \approx 3 \times 10^{-9}$. The proposed EP tests with cold atoms interferometry will measure the differential acceleration between isotopes ${ }^{85} \mathrm{Rb}$ and ${ }^{87} \mathrm{Rb}$, whose difference in composition is unfortunately limited to two neutrons only.

All other experiment proposals aiming at a considerable improvement over the results achieved by rotating torsion balances are to be performed inside a capsule dropped during a balloon flight [17], in a suborbital flight with a sounding rocket [18] or inside a spacecraft in low Earth orbit [19-21].

Theoretical predictions have been made as to what level an EP violation is likely to occur [22-24]. The most recent work [24] indicates that, for test masses made of $\mathrm{Be}-\mathrm{Cu}$ and $\mathrm{Pt}-\mathrm{Ti}$, a violation might occur at a level which is within the reach of current ground tests with rotating torsion balances. However, it is apparent from the speculative nature of these analyses that only a very high accuracy EP test will provide a major breakthrough or-if not-severely constrain the theoretical framework. In this context, tests of composition dependent effects and tests of postNewtonian ones are quantitatively compared to conclude that UFF tests put much more stringent limits than solar system or binary-pulsar test, by several orders of magnitude ([24], Sec. IIIB).

Equivalence principle experiments involving man made test masses do not allow to test it for gravitational self-energy itself because the contribution from gravitational binding energy-mentioned above in relation to the generalized Eötvös parameter (3) - is negligible for artificial bodies. This form of equivalence is often referred to as the Strong Equivalence Principle (SEP) and can be tested only with experiments in which the test masses are celestial bodies - as in the case of the Earth and the Moon falling in the gravitational field of the Sun, the lunar orbit being determined by laser ranging to the Moon. In addition to differing in composition, 
Earth and Moon have a significant component of gravitational binding energy $(4.6 \times$ $10^{-10}$ for the Earth and $1.6 \times 10^{-11}$ for the Moon) whose equivalence can be tested with the current sensitivity of Lunar Laser Ranging (LLR) tests which have reached the level of $10^{-13}$ as tests of the weak equivalence principle [25]. In this sense LLR tests are unique, though they need to be combined with composition dependent tests of laboratory size bodies of Earth-like and Moon-like composition in order to remove their ambiguity as tests of the SEP [12].

The new APOLLO (Apache Point Observatory Lunar Laser-ranging Operation) facility in southern new Mexico [26] will provide-if combined an improved physical model of all perturbations involved - a better determination of the lunar orbit and a more accurate test of the equivalence principle, confirming LLR once more as the most important scientific legacy of the Apollo project to the Moon.

As a test of the equivalence principle, lunar laser ranging is ultimately limited by the non uniformity of the gravity field of the Sun, a limitation expressed by the dimensionless quantity $3 \Delta a / \mathrm{d}$ ( $\Delta a$ being the measurement error in the semimajor axis of the orbit of the Moon around the Earth and d the distance of the Earth-Moon system from the Sun) [27]. A $1 \mathrm{~cm}$ error in semimajor axis (due to $1 \mathrm{~cm}$ accuracy of lunar laser ranging) is consistent with the current level of LLR tests to $10^{-13} ; 1$ order of magnitude improvement is expected with the capability of the APOLLO facility to perform laser ranging to the Moon at $1 \mathrm{~mm}$ level.

The effect of non uniformity of the gravitational field is a real limitation to EP tests with laser ranging because they rely on absolute distance measurements from Earth. However, it is apparent from (1) that EP tests require to measure only differential accelerations - and the displacements they give rise to-of the test masses relative to one another. If artificial test masses are placed inside a spacecraft and differential measurements are performed in situ, displacement sensors are available to measure their relative displacements many orders of magnitude more accurately than by laser ranging from Earth. For testing the equivalence principle there is no need for a very accurate measurement of the absolute distance of the spacecraft from Earth. This is why differential accelerometers to fly inside a spacecraft in low Earth orbit can aim at a far more accurate EP test than lunar laser ranging so as to put General Relativity to the most stringent test ever.

\section{Key instrumental features of the GG experiment in space and their validation on the ground}

The instrument designed to test the Universality of Free Fall (hence the Equivalence Principle) in low Earth orbit is a differential accelerometer with the following properties. The test masses whose relative acceleration effect is the physical quantity to be measured should be co-axial cylinders as concentric as possible, in order to reduce tidal effects, which are also differential but consistent with Newtonian gravity. The test cylinders should be coupled to each other in such a way that accelerations in the plane perpendicular to the symmetry axis are rejected as much as possible if they act in common mode (i.e., the same on both masses), while they are finely sensed if they act on one mass relative to the other (differential accelerations). Finally, this instrument should rotate as fast as possible in order to modulate the expected 
signal at as high frequency as possible, and thus minimize the electronic noise of the transducer (a capacitance bridge in $\mathrm{GG}$ ).

Rotors on the ground need motor and bearings, whose disturbances can be reduced but not eliminated altogether. A satellite in orbit is an isolated system; if it has a cylindrical symmetry, and the symmetry axis is the axis of maximum moment of inertia, once it is set into rotation around this axis at the beginning of the mission (by the launcher and/or by appropriate thrusters), its angular momentum is conserved with no motor or bearings. In GG the spacecraft is co-axial and concentric with the cylindrical test masses inside and the whole system spins together; small angular rotation differences between the outer shell and the instrument inside can be passively compensated, as designed in ([28], Sec. 2.1.2). We therefore have a spacecraft passively stabilized by one-axis rotation (with no need for reaction wheels or other means of attitude control, thus reducing the total satellite mass at launch to only $250 \mathrm{~kg}$ ) and a rotating differential accelerometer (also with cylindrical test masses) without motor and bearings. Note that in GG the payload is weakly coupled to the spacecraft to provide a passive attenuation stage which is effective also in (passively) reducing satellite "nutations" (see [28], Sec. 2.1.2 and Sec. 5.5]).

In space it is therefore possible to realize an isolated rotor made of multiple macroscopic bodies in a nested configuration, all co-rotating, with no motor or bearings, no nearby moving mass anomalies, no local terrain tilt disturbances. This would never be possible on the surface of the Earth. A simple minded way to appreciate the advantages, in terms of low noise, of such an isolated co-rotating system is to think at the diurnal co-rotation of the Earth and its atmosphere, whereby we have no perception of moving (at mid latitudes) at about 1,200 km/h.

The nominal rotation rate chosen for $\mathrm{GG}$ is $2 \mathrm{~Hz}$, which is commonplace for satellites stabilized by one-axis rotation. An EP violation signal from the Earth detected by the differential accelerometer inside the spacecraft would be modulated at this frequency, which is the highest ever attempted in EP experiments and is expected to produce the best reduction of electronic noise (see Fig. 4).

Sensitivity of the accelerometer in 2D (in the plane perpendicular to the spin/symmetry axis) is crucial to the entire experiment design, as shown in [28, 29]. It ensures: (1) fast rotation and high frequency signal simulation with no loss of signal strength; (2) a position of relative physical equilibrium of the test masses uniquely determined by the physical properties of the system (see [29], Fig. 3) around which the effect of a differential force (e.g. an EP violation effect) can be detected; (3) reduction of offset errors due to construction/mounting as the ratio natural-tospin frequency squared; (4) possibility to test the instrument design at 1-g by using the symmetry/rotation axis to suspend the apparatus against local gravity. It can be rigorously demonstrated that none of these properties could be satisfied if the accelerometer is sensitive in one dimension only, like for example the one proposed in [30], because it would be strongly unstable at the spin rates of interest ([31], Ch. 6, p. 228). In addition, with $2 \mathrm{D}$ sensitivity, the amount of measurement data is doubled, with a corresponding noise reduction by a further factor $\sqrt{ } 2$.

The GG spacecraft is shown and briefly described in Fig. 1.

The largest acceleration that the test masses suspended inside the spacecraft are subjected to, is due to the residual air drag acting on its external surface. Air molecules hitting the spacecraft at the relative velocity accelerate it along the orbit (by reducing its kinetic energy and lowering its orbiting altitude); correspondingly, 

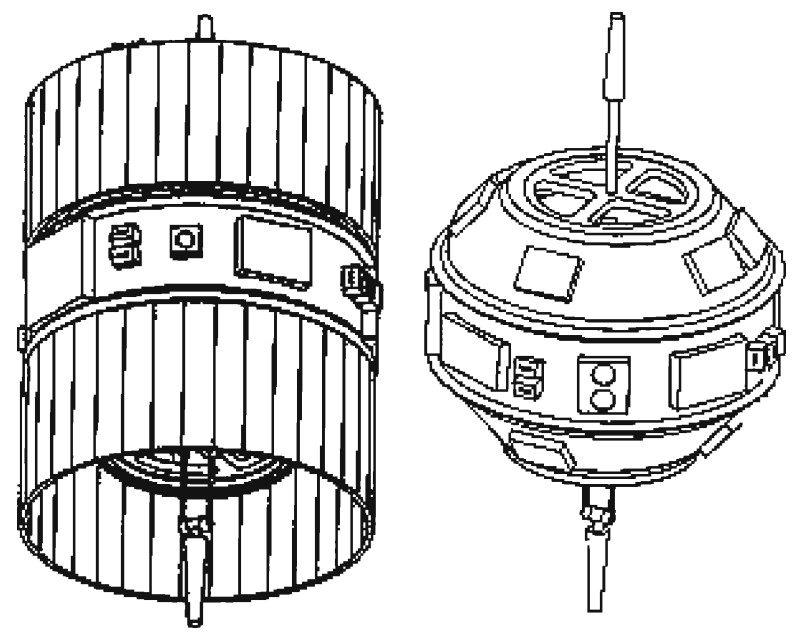

Fig. 1 The GG spacecraft with solar panels (left) and without (right), showing its compact "spinning top"-like shape as designed in [28] to fly in almost circular, almost equatorial orbit at $520 \mathrm{~km}$ altitude around the Earth. The outermost cylinder is $1 \mathrm{~m}$ wide and $1.3 \mathrm{~m}$ high. Nominal rotation rate around the symmetry axis is $2 \mathrm{~Hz}$ and provides attitude stabilization. The symmetry/rotation axis is almost perpendicular to the orbit/equatorial plane. The total mass at launch is $250 \mathrm{~kg}$. The differential accelerometer for testing the Universality of Free Fall is suspended inside the "spinning top", it has the same cylindrical symmetry as the outer skin and is sensitive to differential accelerations in the plane perpendicular to the symmetry/rotation axis, which is close to the equatorial/orbit plane. It would therefore sense the differential acceleration resulting from a breakdown of UFF in the gravitational field of the Earth, and thus test the Equivalence Principle

an equal and opposite inertial acceleration acts on the center of mass of any body suspended inside the spacecraft. A similar effect of comparable magnitude is caused by solar radiation pressure.

In the reference frame of the satellite orbiting around the Earth the gravitational acceleration-only slightly smaller than $9.8 \mathrm{~m} / \mathrm{s}^{2}$ for a low altitude satellite-is exactly balanced by the centrifugal acceleration at its center of mass ("absence of weight"); anywhere away from the center of mass there are non zero residual accelerations ("tidal accelerations"), but they are much smaller than the inertial acceleration caused by air drag which is the dominant one by far. Thus, while on the ground suspended masses must withstand the local gravitational acceleration, inside a spacecraft they must withstand the acceleration caused by air drag and by solar radiation pressure, which are many orders of magnitude smaller than g, particularly in the case of a small and compact satellite like GG. It is therefore apparent that, whatever the physical nature of the suspension used, it can be by far weaker in orbit than on the ground, thus making the natural differential period of the test masses very long, hence the system very sensitive to differential accelerations between them (sensitivity improves as the differential period squared). Last but not the least, the same suspensions provide passive electric grounding of the test masses, an important concern for almost all space missions in fundamental physics employing free test masses. 
In space it is therefore possible to build a differential accelerometer much more sensitive than on the ground (to be equipped with an appropriate lock/unlock mechanism in order to withstand accelerations of several $g$ at launch). Our design is reported in Fig. 2. We notice that the test masses are coupled by the suspensions and also by the capacitance read-out, thus optimizing the instrument for rejecting common mode effects and providing best sensitivity to differential ones. The strong symmetry of the instrument (both azimuthally and top-down) allows us to accommodate two concentric accelerometers, one with test masses of different composition

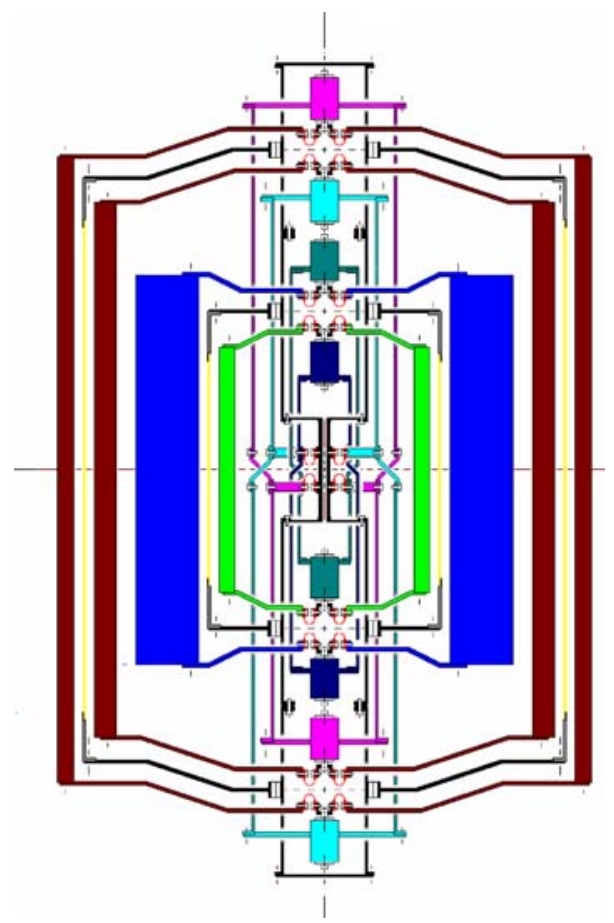

Fig. 2 Section through the spin axis of 2 co-axial, concentric, differential accelerometers for the GG mission. There are four test cylinders (weighing $10 \mathrm{~kg}$ each), one inside the other, all centered at the same point (nominally, the center of mass of the spacecraft) forming two differential accelerometers: the inner one for EP testing (cylinders of different composition; shown in green and blue respectively) and the outer one for zero check (cylinders made of the same material; both shown in brown). In each accelerometer the two test cylinders are coupled to form a beam balance by being suspended at their top and bottom from the two ends of a coupling arm made of two concentric tubes (each tube suspends one test cylinder at each end, which makes it asymmetric top/down; however, the two of them together form a symmetric coupling). All four tubes (two for each coupling arm) are suspended at their midpoints from the same suspension shaft (the longest vertical tube in figure). In all cases the suspensions are $\cup$-shape (or $\cap$-shape) thin strips (shown in red), to be carved out of a solid piece of $\mathrm{CuBe}$. At each connection there are three of them, at $120^{\circ}$ from one another (the planar section in figure shows two for explanatory purposes only). There are capacitance plates (connected to the suspension shaft) for the read-out of differential displacements in between each pair of test cylinders (shown as yellow lines in section). The eight small cylinders drawn along the symmetry axis are inchworms for the fine adjustment of the lengths of the coupling arms in order to center each test mass on the center of mass of the spacecraft. The whole system is symmetric around the spin axis as well as top/down. The two accelerometers are both centered at the center of mass of the spacecraft in order to reduce common mode tidal effects and improve the reliability of the zero check 
(for EP violation detection) and one with test masses of the same composition for zero check. Being concentric, both accelerometers can be centered on the center of mass of the spacecraft so as to ensure the same platform noise and make the zero check reliable.

Note that by using large masses $(10 \mathrm{~kg}$ each) to reduce thermal noise the whole apparatus can be operated at ambient temperature with a total mass of $250 \mathrm{~kg}$ maximum at launch, thus reducing the complexity and the cost of the mission. A low temperature version of the experiment might provide a still greater sensitivity, but with much larger complexity, mass and cost.

According to the GG scientific and industrial study [28], the error budget is compatible-based on realistic requirements-with an EP test in the gravitational field of the Earth to 1 part in $10^{17}$. Since then, the GGG laboratory prototype has been considerably improved thanks to INFN approval as a national experiment, and GG has been included in the National Aerospace Plan of ASI. In 2008, ASI funding allows us to improve the GGG apparatus and to incorporate laboratory results with an advanced industrial study of the GG space experiment. A final mission report will be published in 2009 .

\section{The GGG laboratory prototype}

A sketch of the GGG differential accelerometer, currently located at the INFN lab in Pisa-San Piero a Grado, is shown and briefly described in Fig. 3 (see [32, 33] for details). The test cylinders, shown in green and blue, should be compared to the inner accelerometer of Fig. 2 (same colors). The masses of the test cylinders are the same (10 kg each), though so far in GGG they have the same composition (Al) for cost reasons and because the goal is to test the physical properties and sensitivity of the apparatus. Both instruments are sensitive in the plane perpendicular to the spin/symmetry axis. For GGG this is the horizontal plane; the spin/symmetry axis coincides with the local vertical and the test cylinders are suspended (as described in the caption) to form a beam balance with the beam in the vertical direction and the masses centered on each other. In both cases the capacitance plates are located halfway in between the test masses to measure their relative displacements.

It is very important to stress that local gravity does not play a stabilizing role on the test masses of GGG. It only provides a small "negative" spring which can be used to increase the oscillation period of the test masses relative to each other ([28], Ch. 3), but it has no influence on their position of relative equilibrium, as it has been demonstrated experimentally ([29], Fig. 3). Since no stabilizing effect can be expected in space due to absence of weight, a ground based apparatus where the test mass is instead stabilized by gravity (as, for instance, in [35]) would differ substantially from its counterpart in space.

The similarity between GGG and the inner accelerometer of Fig. 2 is apparent, but we point out the following differences.

(1) In GGG the coupling arm is not located, as in Fig. 2, inside the inner test cylinder with top/down symmetry (for practical mounting reasons). (2) The cardanic suspensions which suspend and couple the test masses (shown in red in Fig. 3) cannot be as weak as in absence of weight, resulting in a natural differential period at present about 40 times smaller than in space, which means that operation in 


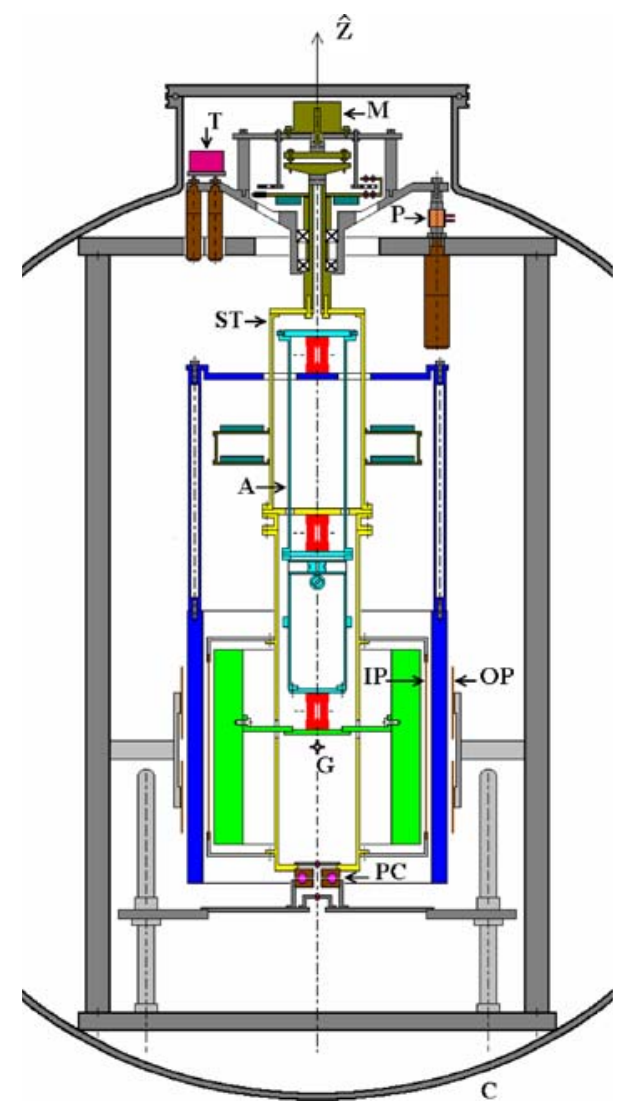

Fig. 3 Section through the spin axis $Z$ of the GGG differential accelerometer inside the vacuum chamber. The drawing is to scale and the inner diameter of the vacuum chamber is $1 \mathrm{~m}$. $C$ vacuum chamber; $M$ motor; $x$ ball bearings; $S T$ suspension tube; $A$ coupling (balance) arm, located inside the suspension tube, with its three laminar cardanic suspensions (in red; the central one suspends the whole weight from the suspension tube, the top and bottom ones suspend outer and inner cylinder respectively from the coupling arm); $G$ center of mass of the two-cylinder system (in blue the outer cylinder, in green the inner one, $10 \mathrm{~kg}$ each). IP are the internal capacitance plates of the differential motion detector, OP are the outer ones for whirl control, PC is the contactless inductive power coupler providing power to the electronics inside the rotor. $T$ and $P$, at the top of the rotor, are the tiltmeter and 3 PZTs (at $120^{\circ}$ from one another-only one shown) for automated control of low frequency terrain tilts

weightlessness conditions would give higher sensitivity to differential accelerations by a factor 1,700 . The mechanical quality and losses of the cardanic suspension, which are crucial to achieve the scientific goal of the mission, have been measured with the GGG apparatus ([34], Fig. 5) demonstrating that the requirements can be met. (3) The motor and bearings shown in Fig. 3 are obviously not needed in space. (4) Appropriate sensor and actuators (tilt meter and PZTs, depicted as T and P in Fig. 3) are used on the ground to reduce disturbances from local terrain tilts, which by changing the inclination of the suspension tube will, because of the nonzero elastic coefficient of the laminar suspensions, produce changes of the inclination 
of the coupling arm (the beam of the balance) resulting in unwanted differential displacements of the test cylinders. Such a closed loop control is not needed in space since the whole satellite is an isolated system.

The capacitance read out of GGG is in all similar to the one required in space. The measured noise is reported in Fig. 4 for various values of the spin rate, demonstrating beyond question that if the signal is modulated at high frequency by spinning the apparatus, the electronic noise is strongly reduced. At $2 \mathrm{~Hz}$ nominal spin frequency of GG the level of noise reported in Fig. 4 is already very close to the requirement of the mission to detect an EP violation of 1 part in $10^{17}$.

Low frequency tilts of the local terrain disturb the test masses of the GGG accelerometer. A closed control loop is applied, using a tilt meter as sensor and PZTs as actuators. Figure 5 shows the results achieved in a 1 week run at the spin frequency of $0.9 \mathrm{~Hz}$. However, though the loop works very well, the "zero" of the tilt meter is not the actual horizontal plane because the tilt meter is temperature dependent. Unless this dependence is characterized and compensated for within the loop, so that the PZTs receive the correct command, "spurious" tilts are in fact applied producing unwanted relative displacements of the test cylinders.

Thermal stabilization of the GGG experiment is very important also because in the current set up the vacuum chamber and the instrumentation is not optimized to reduce diurnal thermal expansion/contraction effects. Figure 6 shows how disturbances on the relative motion of the test cylinders acting over the timescale of 1 day have been reduced by 2 orders of magnitude by an appropriate thermal control loop of the vacuum chamber which has reduced its diurnal temperature variations to $0.02^{\circ} \mathrm{C} / \mathrm{day}$. If then the temperature dependence of the tilt meter is characterized in this range, and compensated for within the control loop before sending the signal to

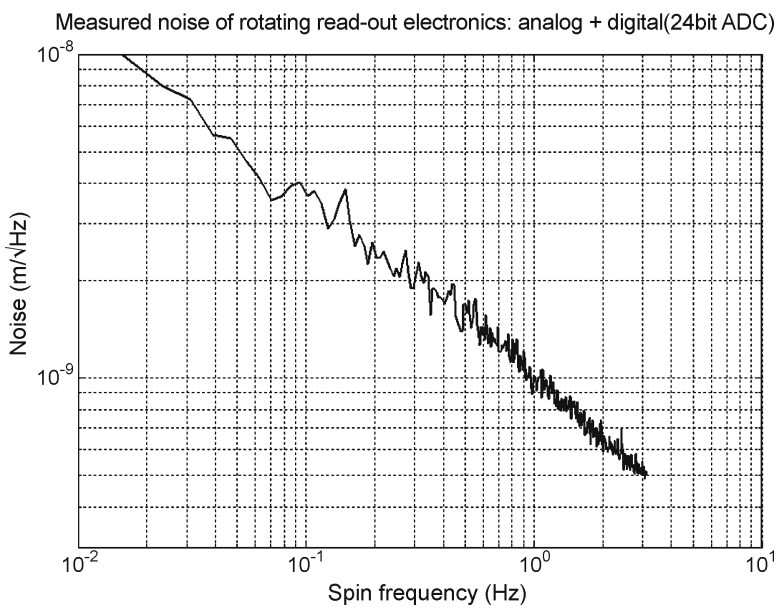

Fig. 4 The read-out of the relative displacements of the test cylinders consists of two capacitance bridges co-rotating with the test masses. The analog output of each bridge is digitized 32 times per turn. Plot shows noise of read-out electronics alone located inside a chamber maintained at $35 \pm$ $0.1^{\circ} \mathrm{C}$. The noise of the digital part (alone) was measured for a few days, sampling at 32 times per spin period, as function of spin frequency (up to $3 \mathrm{~Hz}$ ). The noise of the analog part was measured with the spectrum analyzer. The curve shows the sum of the two. The advantage of spin in reducing electronic noise is apparent 


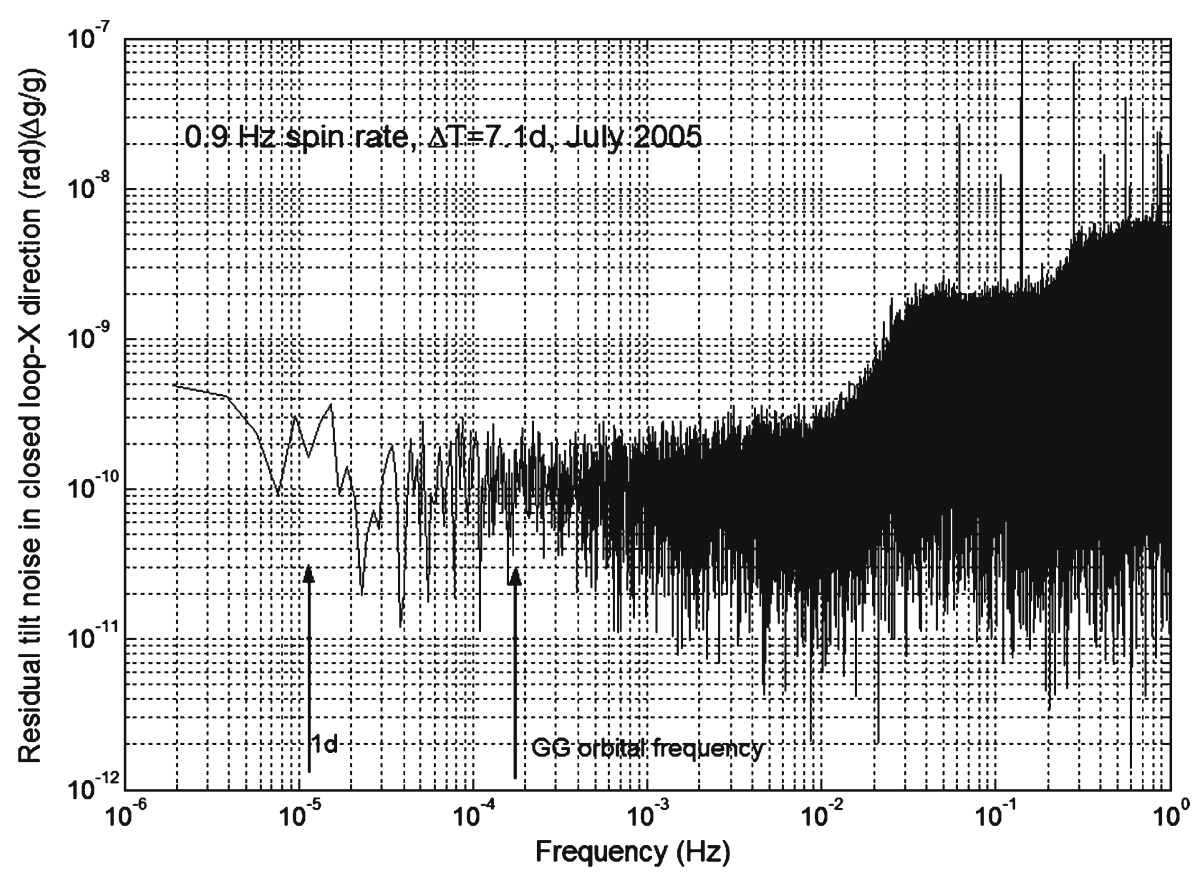

Fig. 5 Fast Fourier Transform of the residual tilt noise after applying tilt control in closed loop for 7.1 days with the GGG apparatus spinning at $0.9 \mathrm{~Hz}$. The test shows that at the low frequencies of interest (the GG orbital frequency and the diurnal frequency), the tilt measurement signal of the sensor used to close the loop can be "zeroed" to a few $10^{-10} \mathrm{rad}\left(10^{-10} \mathrm{rad}\right.$ corresponding, in the current set up, to a relative displacement of the test cylinders of about $\left.0.5 \times 10^{-10}\right) \mathrm{m}$. This is the best result which can be obtained given the sensitivity of the tilt meter used

the PZT actuators, the spurious tilts mentioned above are reduced, the zero of the tilt meter becomes a "true" zero and a corresponding reduction of the low frequency relative displacements of the test masses is expected.

It is worth stressing that a very important temperature induced disturbance known as radiometer effect, which needs laboratory testing for other space experiments in fundamental physics [35], has been demonstrated to be irrelevant in GG and GGG due to the experiment design [36, 37].

In $\mathrm{GG}$ and $\mathrm{GGG}$ the test masses are in supercritical rotation, i.e. they spin at a frequency higher than their natural frequencies of mechanical osci.llations. It is well known that in this case the bodies develop slowly unstable whirl motions at the low frequencies of natural oscillations of the system (the smaller the mechanical losses, the slower the instabilities). Figure 7 shows the algorithm employed in GGG to damp the whirls. The motion of the external mass is observed with two capacitance sensors (indicated as OP in Fig. 3) in $X$ and $Y$ directions in the horizontal plane. The frequency component associated with the whirl motions are isolated from these signals in the frequency domain. Then the time series associated with these motions are reconstructed by an inverse FFT and differentiated to obtain the velocity. A capacitive force equal and opposite to this velocity is applied on the external mass to damp its whirl motion. However since the motion of the mass is not well 


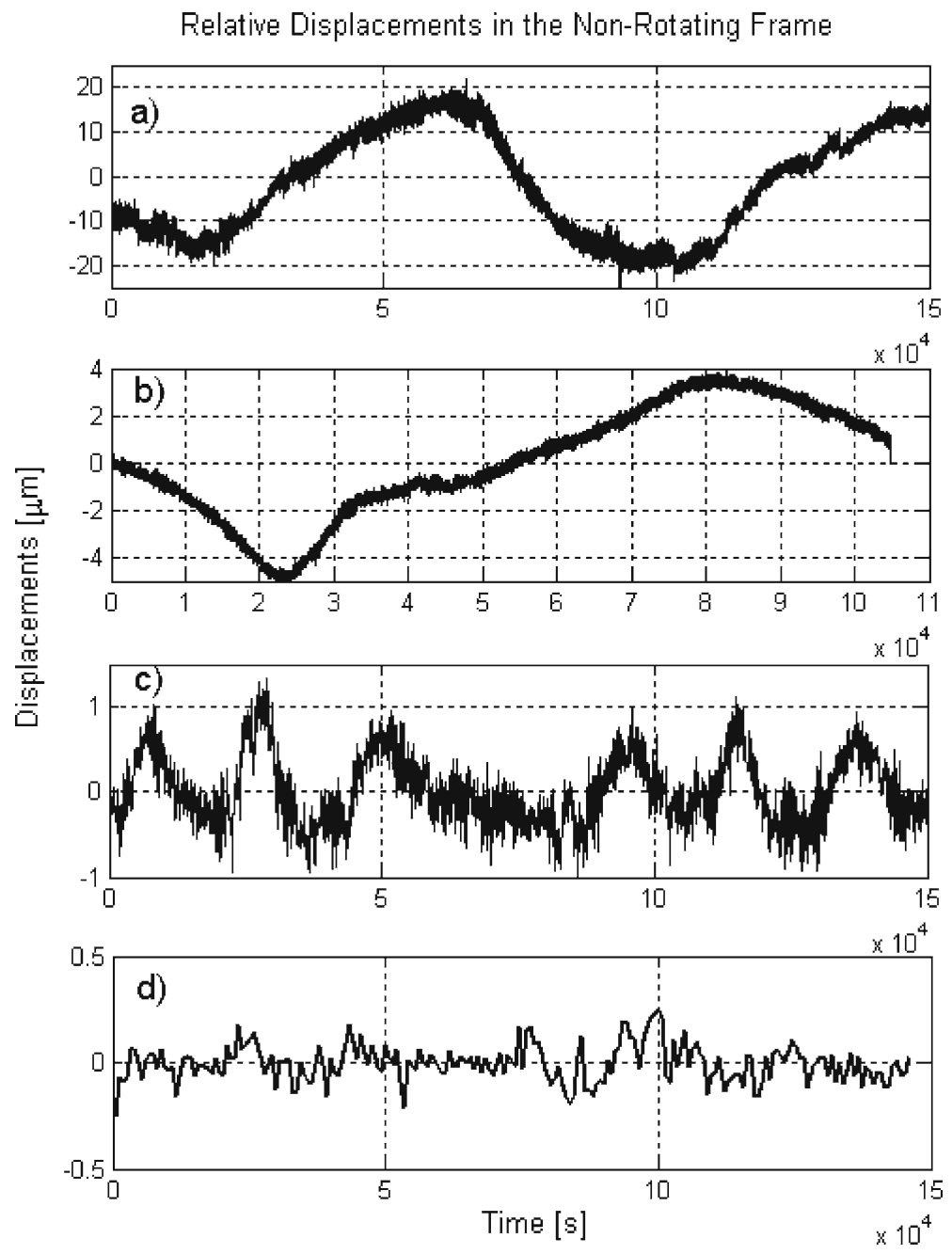

Fig. 6 The figure demonstrates the advantages of thermal stabilization of the vacuum chamber enclosing the GGG experiment. The plots show the relative displacements (in microns) between the centers of mass of the test cylinders in the horizontal plane of the laboratory (once transformed from the rotating reference frame). Each run lasts about 2 days. Diurnal variations are apparent in the two upper plots, while they are no longer visible in the bottom ones; from plot a to plot $\mathbf{d}$ the amplitude of the largest relative displacements has decreased from 20 to $0.2 \mu \mathrm{m}$. The improvement therefore amounts to 2 orders of magnitude. It has been obtained by improving the thermal stability of the chamber. Starting with the run reported in plot $\mathbf{b}$ a thermal stabilization loop of the chamber was implemented and gradually improved during the following runs till reaching the level of $\pm 0.02^{\circ} \mathrm{C} /$ day during the run reported in plot $\mathbf{d}$

reconstructed close to the recent past the signal applied is phase delayed by one beat envelope to ensure that phase errors do not occur. The entire algorithm runs in real time on a moving time series of about $300 \mathrm{~s}$. And thus the frequency components extracted are averaged and updated on this time scale. 
The efficacy of such an active feed-back whirl control at rotation velocities approaching $2 \mathrm{~Hz}$ (typical of GG) has been demonstrated in GGG. The rotor is stable once the whirl has been damped, it can be operated for indefinitely long periods (few months demonstrated) and its sensitivity is not limited by noise associated with whirl damping.

It is worth pointing out that this control loop is in fact similar to the one required in space to compensate for drag, though thrusters are needed as actuators on the outer shell of the spacecraft to reduce the effect of air resistance and make the spacecraft follow the test masses inside it. The lower frequency of drag effect (GG orbital frequency around the Earth) makes the drag compensation loop easier. We are therefore in the unique position of being able to test in the lab a significant part of the GG drag compensation loop, which is a crucial component of the satellite design generally impossible to test on ground. Next step in making the control loop of Fig. 7 closer to the one required in space is to implement it in the rotating rather than in the non rotating frame, since in GG there will be no non rotating parts. The problem has

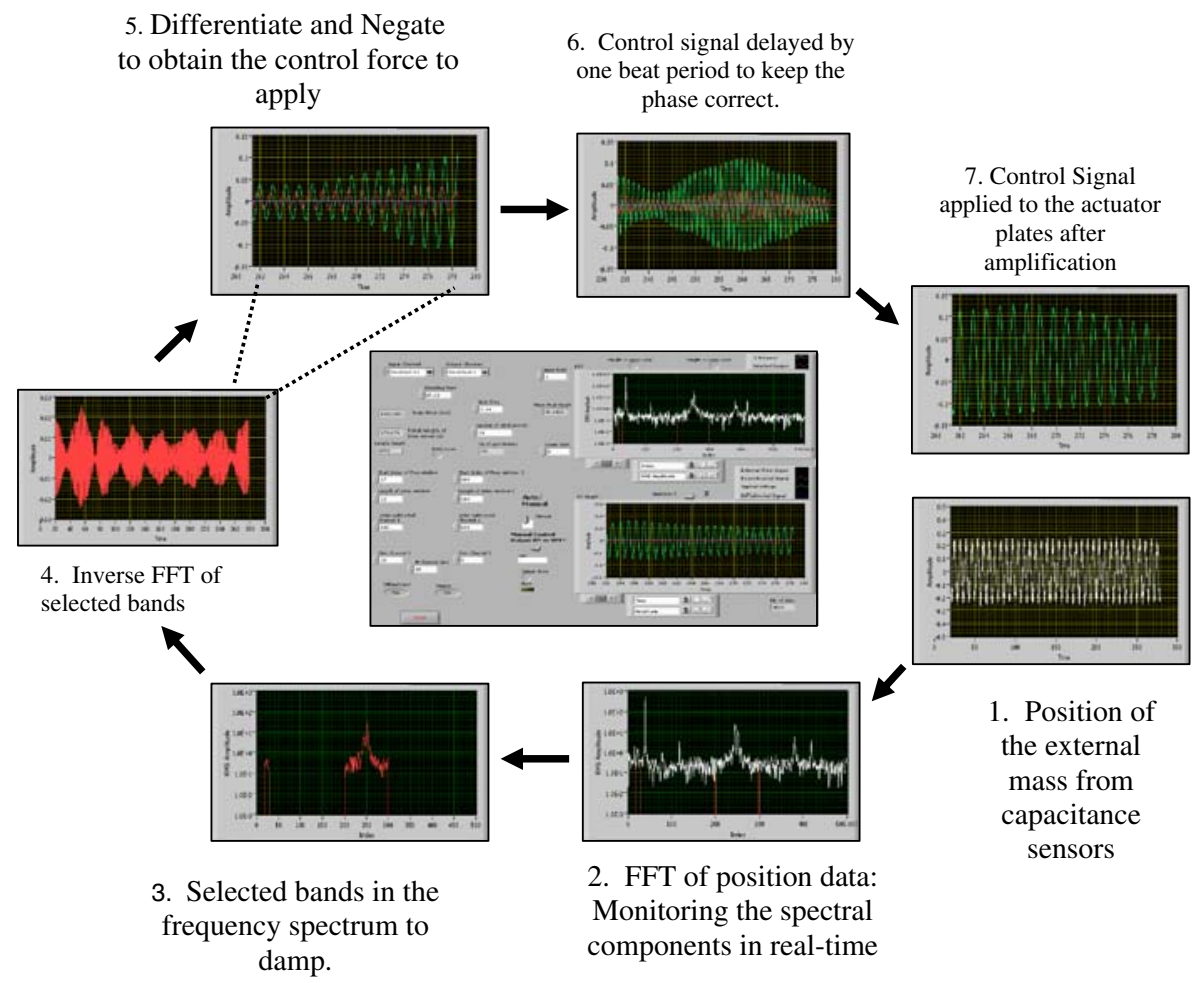

Fig. 7 GGG damping of whirl motions. The active feedback whirl control algorithm for damping selected frequency bands associated with whirl motion has been developed and executed on LabView using standard DAQ cards with 16 bit resolution. The program is re-sizeable to include additional frequency bands. The efficacy of the loop has been demonstrated at rotation velocities approaching $2 \mathrm{~Hz}$ (typical of GG). The rotor is stable once the whirl has been damped, it can be operated for indefinitely long periods (few months demonstrated) and its sensitivity is not limited by noise associated with whirl damping 
been investigated and there are no conceptual obstacle in doing that [38]. In GGG, being it possible and less complicated to implement a non rotating whirl control, the latter choice was made. Moving to a rotating one is not at present a priority but it can and will be done in the future.

Though many other experimental features of the GGG experiment could be discussed, those presented here are some of the most relevant, the most important fact being that GGG allows us to test a system with the same physical properties of the one to fly in space (same number of degrees of freedom, same read-out system, test masses to scale, no stabilizing effect of local gravity on the signal of interest, etc.). This is a unique opportunity for a space mission in fundamental physics which does considerably increase its chances to succeed.

Acknowledgements Thanks are due to ASI (Agenzia Spaziale Italiana) for supporting the small satellite GG at scientific and industrial level, and to INFN (Istituto Nazionale di Fisica Nucleare) for providing us with the laboratory and the funds to develop the GGG experiment.

\section{References}

1. Connecting Quarks with the Cosmos: Eleven Science Questions for the New Century. Board on Physics and Astronomy. The National Academic Press (2003)

2. Report of the Dark Energy Task Force. http://www.nsf.gov/mps/ast/aaac/dark_energy_ task_force/report/detf_final_report.pdf (2006)

3. Bramanti, D., et al.: Galileo and the Universality of Free Fall. In: STEP Symposium, ESA WPP115, 319 (1993)

4. Cajori, F.: Sir Isaac Newton's Mathematical Principles of Natural Philosophy. University of California, Berkeley (1934)

5. Einstein, A.: Über das Relativitätsprinzip und die aus demselben gezogene Folgerungen. Jahrb. Radioaktiv 4, 411 (1907)

6. Will, C.M.: The Confrontation Between General Relativity and Experiment: Living Reviews in Relativity, vol. 9, p. 3. http://relativity.livingreviews.org/Articles/lrr-2006-3/ (2006)

7. Nobili, A.M.: In: Quinn, T.J., Leschiutta, S., Tavella, P. (eds.) Proceedings of the International School of Physics "Enrico Fermi" Course CXLVI Recent Advances in Metrology and Fundamental Constants, pp. 609-652. IOS Press, Amsterdam (2001)

8. Eötvös, R.V., Pekar, D., Fekete, E.: Beitrage zum gesetze der proportionalität von trägheit und gravität. Ann. Phys. 68, 11-66 (1922). doi:10.1002/andp.19223730903

9. Roll, P.G., Krotkov, R., Dicke, R.H.: The equivalence of inertial and passive gravitational mass. Ann. Phys. 26, 442-517 (1964). doi:10.1016/0003-4916(64)90259-3

10. Braginsky, V.B., Panov, V.I.: Verification of the equivalence of inertial and gravitational mass. Sov. Phys. JEPT 34, 463-466 (1972)

11. Su, Y., Heckel, B.R., Adelberger, E.G., Gundlach, J.H., Harris, M., Smith, G.L., Swanson, H.E.: New tests of the universality of free fall. Phys. Rev. D Part. Fields 50, 3614-3636 (1994). doi:10.1103/PhysRevD.50.3614

12. Baeßler, S., Heckel, B.R., Adelberger, E.G., Gundlach, J.H., Schimidt, U., Swanson, H.E.: Improved test of the equivalence principle for gravitational self-energy. Phys. Rev. Lett. 83, 3585 (1999). doi:10.1103/PhysRevLett.83.3585

13. Schlamminger, S., Choi, K.-Y., Wagner, T.A., Gundlach, J.H., Adelberger, E.G.: Test of the equivalence principle using a rotating torsion balance. Phys. Rev. Lett. 100, 041101 (2008)

14. Carusotto, S., Cavasinni, V., Mordacci, A., Perrone, F., Polacco, E., Iacopini, E., Stefanini, G.: $g$ universality with a Galileo type experiment. Phys. Rev. Lett. 69, 1722 (1992)

15. Dimopoulos, S., Graham, P.W., Hogan, J.M., Kasevich, M.A.: Testing general relatività with atom interferometry. Phys. Rev. Lett. 98, 111102 (2007). doi:10.1103/PhysRevLett.98.111102

16. Peters, A., Chung, K.Y., Chu, S.: Measurement of gravitational acceleration by dropping atoms. Nature 400, 849 (1999). doi:10.1038/23655 
17. Iafolla, V., Lorenzini, E.C., Milyukov, V., Nozzoli, S.: Methodology and instrumentation for testing the weak equivalence principle in atmospheric free fall. Rev. Sci. Instrum. 69, 4146 (1998). doi:10.1063/1.1149261

18. Reasenberg, R.D.: A Sounding Racket to Test the WEP, presented at Q2C3 International Workshop, Airlie Center, Virginia USA. http://funphysics.jpl.nasa.gov/Q2C3/program. html (2008)

19. $\mu$ CROSCOPE Website. http://www.onera.fr/dmph/accelerometre/index.html

20. "Galileo Galilei" (GG) Website. http://eotvos.dm.unipi.it

21. STEP Website. http://einstein.stanford.edu/STEP/step2.html

22. Fischbach, E., Krause, D.E., Talmadge, C., Tadic, D.: Higher order weak interactions and the equivalence principle. Phys. Rev. D Part. Fields 52, 5417 (1995). doi:10.1103/PhysRevD.52.5417

23. Damour, T., Polyakov, A.M.: Runaway dilaton and equivalence principle violations. Gen. Relativ. Gravit. 26, 1171 (1994). doi:10.1007/BF02106709

24. Damour, T., Piazza, F., Veneziano, G.: Violations of the equivalence principle in a dilatonrunaway scenario. Phys. Rev. D Part. Fields 66, 046007 (2002). doi:10.1103/PhysRevD.66.046007

25. Williams, J.G., Turyshev, S.G., Boggs, D.H.: Progress in lunar laser ranging tests of relativistic gravity. Phys. Rev. Lett. 93, 261101 (2004). doi:10.1103/PhysRevLett.93.261101

26. Murphy, T.W. Jr., Michelson, E.L., Orin, A.E., Adelberger, E.G., Hoyle, C.D., Swanson, H.E., Stubbs, C.W., Battat, J.E.: APOLLO: Next-Generation lunar laser ranging. San Diego, UCSD. Int. J. Mod. Phys. D16(12a), 2127 (2007)

27. Nobili, A.M., Comandi, G.L., Bramanti, D., Doravari, S., Lucchesi, D.M., Maccarrone, F.: Limitations to testing the equivalence principle with satellite laser ranging. Gen. Relativ. Gravit. 40, 1533-1554 (2008). http://eotvos.dm.unipi.it/documents/generalpapers/nobili.pdf

28. "Galileo Galilei" (GG) Phase A Report, Nobili A.M., et al.: ASI (Agenzia Spaziale Italiana), 1st edn. November 1998, 2nd edn. (January 2000). http://eotvos.dm.unipi.it/ggweb/phaseA/ (January 2000)

29. Nobili, A.M., Comandi, G.L., Doravari, S., Maccarrone, F., Bramanti, D., Polacco, E.: Experimental validation of a high accuracy test of the equivalence principle with the small satellite "Galileo Galilei". Int. J. Mod. Phys. D 16(12a), 2259-2270 (2007). doi:10.1142/S0218271807011590

30. Chapman, P.K., Hanson, A.J.: Proceedings of the Conference on Experimental Tests of Gravitational Theories. Cal. Tech. JPL TM 33-499, 228 (1970)

31. Den Hartog, J.P.: Mechanical Vibrations. Dover, New York (first published in 1934) (1985)

32. Comandi, G.L., Chiofalo, M.L., Toncelli, R., Bramanti, D., Polacco, E., Nobili, A.M.: Dynamical response of the Galileo Galilei on the ground rotor to test the equivalence principle: theory, simulation, and experiment. I. The normal modes. Rev. Sci. Instrum. 77(034501), 1-15 (2006)

33. Comandi, G.L., Toncelli, R., Chiofalo, M.L., Bramanti, D., Nobili, A.M.: Dynamical response of the Galileo Galilei on the ground rotor to test the equivalence principle: Theory, simulation, and experiment. II. The rejection of common mode forces. Rev. Sci. Instrum. 77(034502), 1-10 (2006)

34. Comandi, G.L., Nobili, A.M., Bramanti, D., Toncelli, R., Polacco, E., Chiofalo, M.L.: "Galileo Galilei (GG) on the Ground-GGG": experimental results and perspectives. Phys. Lett. A 318, 213-222 (2003). doi:10.1016/j.physleta.2003.07.021

35. Carbone, L., Cavalleri, A., Ciani, G., Dolesi, R., Hueller, M., Tombolato, D., Vitale, S., Weber, W.J.: Thermal gradient-induced forces on geodesic reference masses for LISA. Phys. Rev. D Part. Fields Gravit. Cosmol. 76, 102003-1 (2007). doi:10.1103/PhysRevD.76.102003

36. Nobili, A.M., Bramanti, D., Comandi, G.L., Toncelli, R., Polacco, E., Catastini, G.: Radiometer effect in space missions to test the equivalence principle. Phys Rev, D. Rapid Commun. 63, 101101(R) (2001)

37. Nobili, A.M., Bramanti, D., Comandi, G.L., Toncelli, R., Polacco, E.: Radiometer effect in the $\mu$ SCOPE space mission. Astron. 7, 521-529 (2002). doi:10.1016/S1384-1076(02)00175-6

38. Nobili, A.M., Bramanti, D., Polacco, E., Catastini, G., Anselmi, A., Portigliotti, S., Lenti, A., di Giamberardino, P., Monaco, S., Ronchini, R.: Evaluation of a proposed test of the weak equivalence principle using earth-orbiting bodies in high-speed co-rotation: reestablishing the physical bases. Class. Quantum Gravity 16, 1463-1470 (1999). doi:10.1088/0264-9381/16/4/032 